\section{Dynamic range and linearity trade-off in detectors for interferometric radiometers}

F. Torres, N. Duffo, I. Corbella, A. Camps, M. Vall.llossera and L. Sagués

The dynamic range and error performance of the diode power detector used to denormalise the digital correlations in interferometric radiometers is analysed by means of a second-order model of the diode response. This gives an easy method to establish system dynamic range as a trade-off between both the error contribution of measurement uncertainty and diode nonlinearity. The method is illustrated by analysing the power measurement system of the MIRAS-SMOS instrument.

Introduction: This Letter presents a simplified trade-off analysis of the dynamic range and error performance of the power measurement system (PMS) in each microwave imaging radiometer with aperture synthesis (MIRAS) [1], which is the single payload of the ESA-SMOS mission [2]. MIRAS consists of a Y-shape interferometric radiometer formed by 69 receivers placed along the arms. Cross-correlation of the signals collected by all receiver pairs gives a sample of the so-called visibility function, and the brightness temperature map is obtained, in a first approximation, by an inverse Fourier transform. Since the instrument uses 1-bit digital correlators, it actually measures normalised cross-correlations. Hence, the equivalent system temperature at the input of each receiver must be measured in order to denormalise each visibility sample prior to inversion [3]. These measurements are performed by means of a PMS placed in each receiver in the signal path prior to the correlator unit.

Four point measurement technique: A simplified block diagram of a PMS is shown in Fig. 1. When an equivalent system temperature $T_{s y s}$ is driven to its input, the measured voltage is given by

$$
V=V_{o f f}+G T_{s y s}=V_{o f f}+G\left(T_{e x t}+T_{r}\right)
$$

where a linear model of the diode power detector has been taken into account, $T_{r}$ is the receiver equivalent noise temperature, and $T_{e x t}$ stands for the equivalent external temperature. In the measurement mode, this one is given by the equivalent antenna temperature $T_{A}$, while in calibration mode it is given by the so-called COLD and HOT temperatures $T_{C}$ and $T_{H}$. The overall system gain can be switched between two values $G$ and $G / L(L>1)$ by means of an attenuator placed in the signal path at a point that it does not affect $T_{r}$ [4]. For the different combinations of $T_{C}, T_{H}$ and $L$ the following set of voltage measurements are obtained:

$$
\begin{array}{ll}
V_{1}=V_{\text {off }}+G\left(T_{C}+T_{r}\right) & V_{2}=V_{\text {off }}+G\left(T_{H}+T_{r}\right) \\
V_{3}=V_{\text {off }}+\frac{G}{L}\left(T_{C}+T_{r}\right) & V_{4}=V_{\text {off }}+\frac{G}{L}\left(T_{H}+T_{r}\right)
\end{array}
$$

From (2) the PMS unknown parameters $V_{\text {off }}$ and $G$ are readily obtained as

$$
V_{\text {off }}=\frac{V_{2} V_{3}-V_{1} V_{4}}{\left(V_{2}-V_{4}\right)-\left(V_{1}-V_{3}\right)} \quad \text { and } \quad G=\frac{V_{2}-V_{1}}{T_{H}-T_{C}}
$$

and the required equivalent system temperature $T_{s y s A}$ is estimated as:

$$
T_{s y s A}=T_{A}+T_{r}=\frac{V_{A}-V_{o f f}}{V_{2}-V_{1}}\left(T_{H}-T_{C}\right)
$$

where $V_{A}$ is the PMS voltage reading in measurement mode. The main advantage of this approach lies in the fact that $T_{H}$ and $T_{C}$ appear exclusively in differential mode. This is specially important because $T_{H}$ and $T_{C}$ are injected by a noise source (NS) simultaneously to a large set of receivers via a noise distribution network (NDN) [1, 3] (Fig. 1). $T_{H}$ and $T_{C}$ are also injected through the same NDN to a noise injection radiometer (NIR) in order to have absolute knowledge of both $T_{H}$ and $T_{C}$. Hence, noise contribution of the NDN itself is removed because of the differential mode $\left(T_{H}-T_{C}\right)$.

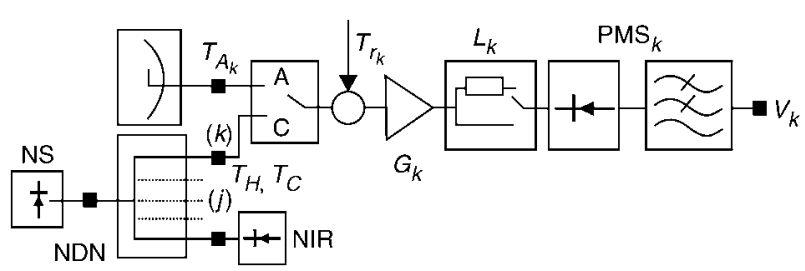

Fig. 1 Simplified block diagram of PMS and two-level noise injection network

Effect of measurement uncertainties: There are two main error sources of $T_{\text {sysA }}$ : PMS nonlinearity and measurement uncertainty in $V, T_{H}$, and $T_{C}$. The impact of the measurement uncertainty is directly related to system dynamic range, which can be defined as the product $D R=Y \cdot L$, where $Y$ is the ratio of system temperatures in HOT and COLD modes: $Y=\left(T_{H}+T_{r}\right) /\left(T_{C}+T_{r}\right)$. Note that the actual value $L$ of the attenuator is not required to estimate $T_{s y s A}$, however it has a large impact in the estimation of $V_{\text {off }}$, since the values into parenthesis in (3) tend to zero as $L$ tends to $1(0 \mathrm{~dB})$. Fig. $2 a$ gives the standard deviation of the fractional error in the estimation of $T_{s y s A}\left(\sigma_{T s y s A}\right.$ in $\%$ ) against $L$ (x-axis) and $Y$ (parametric curves for $Y=2,4,8,16)$. The fractional standard deviation of the PMS voltage readings is $\sigma_{V}=$ $0.1 \%$ and the uncertainty in the calibration temperatures are $\sigma_{T h, T c}=$ $0.1 \%$. The plots in Fig. $2 b$ do not take into account diode nonlinearity which is discussed in the following Sections. With this assumption, it is clear that both $Y$ and $L$ should be as large as possible in order to reduce the effect of measurement uncertainty.
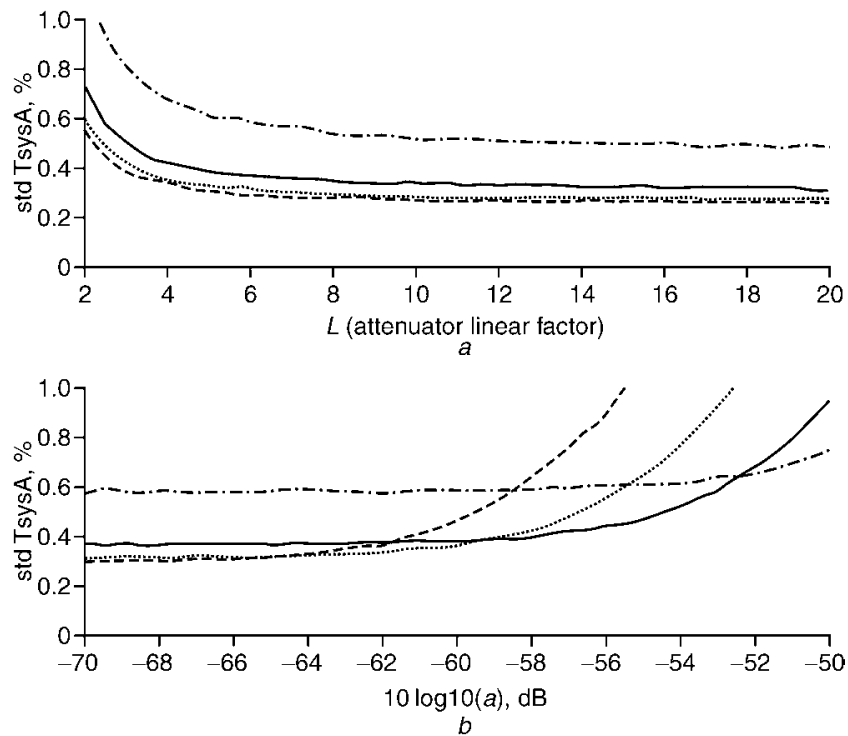

Fig. 2 Error in estimation of $T_{\text {sys } A}$ against $L$ and linearity

a Against $L$

$b$ Against linearity

$\sigma_{V}=\sigma_{T h, T c}=0.1 \%, L=6$

$Y=2(\cdot-\cdot-)$

$Y=4 \quad(-)$

$Y=8 \quad(\cdots)$

$Y=16(----)$

Power detector characterisation: Power measurement is implemented by means of a Schotky diode detector followed by a lowpass filter as integrator. To test the method, four detectors have been characterised. Voltage readings range from 170 to $1150 \mathrm{mV}$ for equivalent system temperatures ranging from 475 to $3950 \mathrm{~K}$. Fig. $3 a$ gives the error of measured data when fitted to a linear model (1) - in a least squares sense. The effect of nonlinearity is clearly seen, giving a standard deviation from the linear model of $\sim 0.4 \%$. To asses the impact of nonlinearity in the detector performance, data has been fitted to a second-order model given by

$$
V_{k}=V_{o f f k}+G_{k} T_{s y s}+a_{k} T_{s y s}^{2}
$$

The parameter $a_{k}$ gives the degree of nonlinearity of the PMS numbered ' $k$ '. Fig. $3 b$ represents the error of measured data in relation to the second-order model. Now the standard deviation of the error is 
$\sim 0.06 \%$, clearly due to measurement uncertainty. Hence, PMS nonlinearity can be well represented by a second-order model with mean $\langle a\rangle=1.6 \cdot 10^{-6} \mathrm{mV} / \mathrm{K}^{2}, \quad\langle G\rangle=0.292 \mathrm{mV} / \mathrm{K}, \quad\left\langle V_{\text {off }}\right\rangle=75.82 \mathrm{mV}$ and standard deviation $\sigma_{a}=3.0 \cdot 10^{-7} \mathrm{mV} / \mathrm{K}^{2}, \sigma_{G}=0.032 \mathrm{mV} / \mathrm{K}$ and $\sigma_{\text {Voff }}=6.57 \mathrm{mV}$. Data from PMS2 have been discarded since it clearly presents a bad performance.
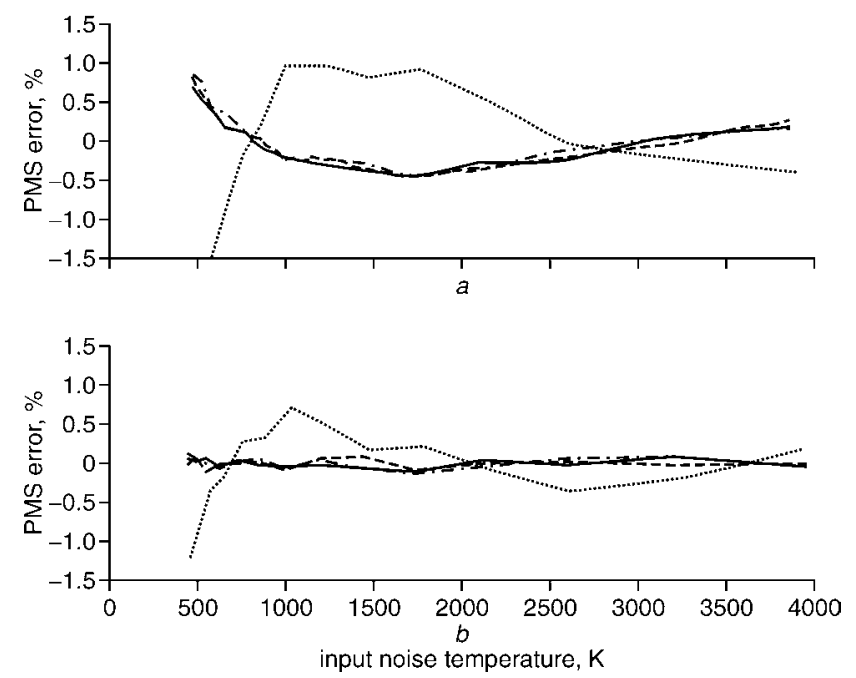

Fig. 3 PMS error: linear model and quadratic model

$a$ Linear model

$b$ Quadratic model

Measured units:

$\mathrm{PMS}_{1}(-)$

$\mathrm{PMS}_{2}(\cdots)$

$\mathrm{PMS}_{3}(\cdot-\cdot-)$

$\mathrm{PMS}_{4}(--)$

Effect of nonlinearity and dynamic range trade-off: Fig. $2 b$ shows the standard deviation of the estimated system temperature, against the second-order effect setting the attenuator to $L=6$ to keep a moderate dynamic range. The actual PMS voltage readings have been simulated by means of the second-order model, while the estimated $T_{s y s A}$ has been obtained through the linear model in (2). The $x$-axis represents the effect of the second-order parameter $a_{k}-(5)$ ranging from -70 to $-50 \mathrm{~dB}$, being the actual value for the measured set of samples of $10 \log \left(a_{k}\right)=-58 \mathrm{~dB}$. For low values of $a_{k}$ Fig. $2 b$ shows that the dominant contribution to the error in $T_{s y s A}$ is given by measurement uncertainty (the same value shown in Fig. $2 a$ for $L=6$ ). However, as nonlinearity increases, the effect of nonlinearity becomes the dominant effect, which is highly dependent on the value of $Y$. In this particular case, a trade-off value for $Y$ that minimises the error is given by $Y=4$. Optimum dynamic range becomes $D R=24(13.8 \mathrm{~dB})$ giving equivalent input temperatures at the receiver front end of $T_{\text {sysmin }}=78 \mathrm{~K}$ and $T_{\text {sysmax }}=1872 \mathrm{~K}$ with associated PMS voltage readings of $V_{\min }=98.6 \mathrm{mV}$ and $V_{\max }=628.05 \mathrm{mV}$.

Conclusion: A second-order model of a diode power detector provides an easy way to determine the optimum system dynamic range in the MIRAS-SMOS interferometric radiometer as a trade-off between both the error contribution of measurement uncertainty and diode nonlinearity.

Acknowledgments: This work is financed by the Spanish MCYT and EU FEDER funds under project TIC2002-04451-C02-01 and EADS CASA Espacio, under the SMOS PLM project.

(C) IEE 2003

Electronics Letters Online No: 20031164

18 September 2003 DOI: 10.1049/el:20031164

F. Torres, N. Duffo, I. Corbella, A. Camps and M. Vall.llossera (Department of Signal Theory and Communications, Polytechnic University of Catalonia, Barcelona, Spain)

E-mail: xtorres@tsc.upc.es

L. Sagués (Space Department, Mier Comunicaciones SA, La Garriga, Spain)

\section{References}

1 MARTIN-NEIRA, M., and GOUTOULE, J.M.: 'A two-dimensional aperture synthesis radiometer for soil moisture and ocean salinity observations', ESA Bull., 1997, 92, pp. 95-104

2 SILVESTRINI, P., et al.: 'ESA's second Earth Explorer Opportunity Mission: The Soil Moisture and Ocean Salinity Mission-SMOS', IEEE Geosci. Remote Sens. Newsl., March 2001

3 TORRES, F., et al.: 'On board phase and modulus calibration of large aperture synthesis radiometers: study applied to MIRAS', IEEE Trans. Geosci. Remote Sens., 1996, 34, (4), pp. 1000-1009

4 PIIRONEN, P.: 'PMS offset determination using an IF attenuator'. Technical Note 14629/00/NL/SF, ESA-ESTEC, Holland, June 2002 